\title{
ABSCESSO ESPLÊNICO CAUSADO POR SALMONELLA
}

\section{SALMONELLAL ESPLENIC ABSCESS}

\author{
Bruno von Glen Herkenhoff, AsCBC-RJ' ${ }^{1}$ [Ludmila Godoy dos Santos Ferreira ${ }^{2}$; Maristela Cavedagne ${ }^{3}$; \\ Renato Manganelli Salomão,TCBC-RJ ${ }^{4}$; Acyolli Moreira Maia, TCBC-RJ ${ }^{5}$; Antonio Carlos Iglesias, TCBC-RJ ${ }^{6}$
}

\begin{abstract}
RESUMO: Objetivo: Relatar as características demográficas, clínicas, diagnóstica e terapêutica de pacientes com abscesso esplênico (AE) causado por Salmonella. Método: Análise retrospectiva de dados de pacientes atendidos no Serviço de Cirurgia Geral e Aparelho Digestivo do Hospital Universitário Gaffrée-Guinle no período de janeiro de 2001 a dezembro de 2005, a estes se incluiu um caso tratado em outro hospital em época anterior. Resultados: Dentre 4823 pacientes recentemente atendidos, dois apresentaram AE causado por Salmonella enteritidis, enquanto o caso mais antigo o agente responsável foi a Salmonella typhi. Todos eram homens, com idade média de 45 anos. Em nenhum deles foi identificada condição predisponente à formação do abscesso; os exames de imagem foram capazes de diagnosticar o AE. Todos foram tratados por esplenectomia e antibioticoterapia e evoluíram para cura. Conclusão: A Salmonella, apesar de infrequente, pode ser o agente causal do AE. Caracteristicamente os abscessos eram grandes e apresentavam material necrótico em seu interior. Nesta condição, a esplenectomia associada a antibioticoterapia mostrou-se eficaz no tratamento (Rev. Col. Bras. Cir. 2006; 33(3): 0-0).
\end{abstract}

Descritores: Baço; Abscesso; Esplenopatias; Infecções por Salmonella; Esplenectomia.

\section{INTRODUÇÃO}

O abscesso esplênico (AE) é uma doença rara, com menos de 400 casos descritos desde o início do século XX até o final dos anos $80^{1}$. A partir de então, um número crescente de casos isolados e de pequenas séries institucionais vem sendo publicado, em parte, pela mudança no perfil dos pacientes que desenvolvem AE. Desta forma, o AE passou a estar associado especialmente a condições de imunossupressão, tais como a Síndrome da Imunodeficiência Adquirida (SIDA), tratamento quimioterápico antineoplásico mais agressivo e a terapêutica imunossupressora pós-transplante de órgãos ${ }^{1-4}$, apesar destas associações, ainda hoje, por se tratar de doença infreqüente e de apresentação clínica pouco característica, o AE permanece como um desafio na prática médica. O retardo no diagnóstico e no tratamento do AE implica em elevada taxa de mortalidade ${ }^{2,3}$.

A maioria dos AE são causados por Staphylococcus aureus, estreptococos ou bacilos gram negativos $^{1,5-7}$. No entanto, tem sido observado aumento no número de casos cujos micro-organismos responsáveis podem ser germes anaeróbios, Pseudomonas $s p$ ou diferentes tipos de fungos ${ }^{5,7}$. Embora na era pré-antibiótico cerca de
1,5 a $1,8 \%$ dos pacientes com febre tifóide apresentassem $\mathrm{AE}$, hoje isto é uma complicação incomum da infecção pela Salmonella typhi. Entretanto, a Salmonella $s p$ ainda responde por cerca de 2 a $20 \%$ dos AE relatados na literatura médica; o sorotipo Typhi é o mais comumente encontra$\mathrm{do}^{1,2,5,6,8,9,15}$.

O objetivo deste trabalho é relatar as características demográficas, clínicas, diagnóstica e a terapêutica de dois casos de AE por Salmonella enteritidis e um outro por Salmonella typhi em pacientes sem qualquer tipo de fator predisponente.

\section{MÉTODO}

Foram avaliados retrospectivamente 4823 prontuários de pacientes internados na $6^{\mathrm{a}}$ Enfermaria do Hospital Universitário Gaffrée-Guinle no período compreendido entre janeiro de 2001 e dezembro de 2005. Aos achados obtidos nesta revisão incluiu-se um caso de $\mathrm{AE}$ tratado por um dos autores (AMM). O diagnóstico de AE baseou-se na presença de pelo menos um dos seguintes achados: (1) confirmação histopatológica de abscesso esplênico em espécie cirúrgica ou material obtido por punção do baço; (2) ob-

1. Residente do Serviço de Cirurgia Geral e Aparelho Digestivo - $6^{\text {a }}$ Enfermaria do Hospital Universitário Gaffrée-Guinle - Universidade Federal do Estado do Rio de Janeiro -UNIRIO.

2. Ex-Interna da Disciplina de Clínica Cirúrgica "A" da Escola de Medicina e Cirurgia da Universidade Federal do Estado do Rio de Janeiro -UNIRIO.

3. Professora Auxiliar da Disciplina de Clínica Cirúrgica "A" da Escola de Medicina e Cirurgia ;Cirurgiã do Serviço de Cirurgia Geral e Aparelho

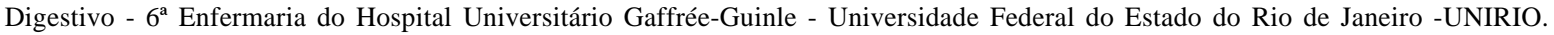

4. Professor Adjunto da Disciplina de Clínica Cirúrgica "A" da Escola de Medicina e Cirurgia. Cirurgião do Serviço de Cirurgia Geral e Aparelho Digestivo - $6^{a}$ Enfermaria do Hospital Universitário Gaffrée-Guinle - Universidade Federal do Estado do Rio de Janeiro -UNIRIO.

5. Professor Titular do Departamento de Cirurgia da Universidade Federal Fluminense.

6. Professor Titular da Disciplina de Clínica Cirúrgica "A" da Escola de Medicina e Cirurgia. Chefe do Serviço de Cirurgia Geral e Aparelho Digestivo

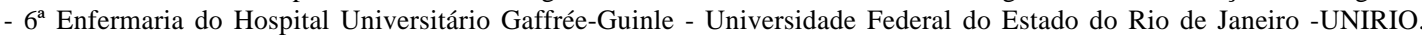

Recebido em

Aceito para publicação em

Conflito de intersses: nenhum

Fonte de financiamento: nenhum

Trabalho realizado no Hospital Universitário Gaffrée-Guinle - Escola de Medicina e Cirurgia - Universidade Federal do Estado do Rio de Janeiro (UNIRIO). 
servação de material purulento no baço durante a operação; e (3) na presença de manifestações clínicas e de diagnóstico por tomografia computadorizada compatíveis. As informações coletadas incluem: aspectos demográficos, clínicos (história, sinais e sintomas, exame físico, comorbidades), laboratoriais e de exames de imagem, bem como as informações referentes ao tratamento e a evolução clínica dos pacientes.

\section{RESULTADOS}

O AE foi diagnosticado em $2(0,04 \%)$ dos 4823 pacientes constantes da avaliação retrospectiva; ambos eram do sexo masculino, assim como o terceiro paciente incluído neste estudo. A idade dos pacientes foi 28,40 e 67 anos. Dois pacientes apresentaram em comum viagem pelas regiões norte e nordeste do país, durante ou logo após a qual teve início a sintomatologia. O tempo decorrido entre o início dos sintomas e o diagnóstico do AE foi de 11,33 e 38 dias. A febre, a dor abdominal em hipocôndrio esquerdo (2/3) ou difusa (1/3), a diarréia e o vômito estiveram presentes em todos os pacientes. Em nenhum deles foi identificada qualquer tipo de condição predisponente ao desenvolvimento do AE. Todos apresentavam diminuição do murmúrio vesicular no terço inferior do hemitórax esquerdo, dois referiam dor à palpação profunda em quadrante superior esquerdo, enquanto o outro apresentava dor abdominal difusa com sinais de irritação peritoneal e distensão abdominal. Este último evoluiu para choque séptico e insuficiência respiratória nas primeiras horas de internação.

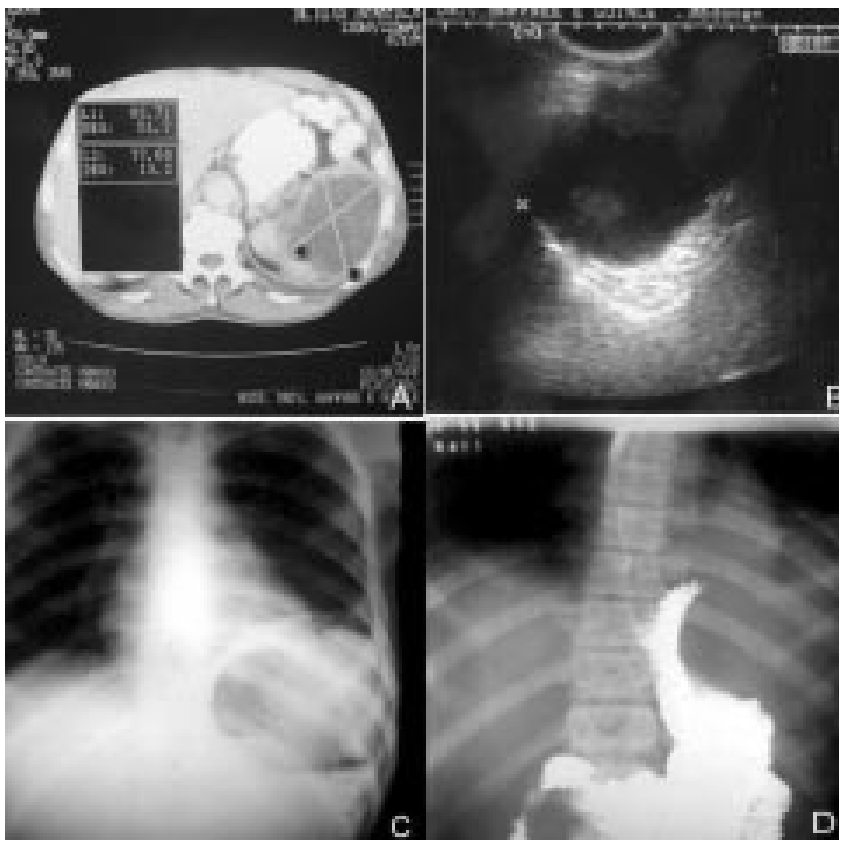

Figura 1 - Em A, tomografia computadorizada evidencia AE de grande volume; em B aspecto ultrassonográfico do AE em cuja cavidade é possivel observar material necrótico. Em C a radiografia simples do abdome mostra imagem hidro-aérea localizada em hipocôndrio esquerdo, cujo exame contrastado do estômago (D) revela compressão e rechaço da grande curvatura pelo $A E$.
A leucocitose se fez presente em dois pacientes, com respectivamente 26700 e 30000 leucócitos $/ \mathrm{mm}^{3}$; o terceiro, apesar de não haver leucocitose (9250 leucócitos/ $\mathrm{mm}^{3}$ ), apresentava-se com 18 bastões e granulações grosseiras nos neutrófilos. A radiografia o tórax evidenciou derrame pleural à esquerda e elevação da hemi-cúpula diafragmática correspondente nos três pacientes. Em um dos pacientes a ultra-sonografia (US) revelou lesão única no polo superior do baço de características císticas com inúmeros debris em seu interior, medindo $8,5 \mathrm{~cm}$ de diâmetro não circundada por tecido esplênico em sem limite proximal. $\mathrm{O}$ outro paciente foi submetido à tomografia computadorizada (TC) do abdome que identificou AE solitário unilocular com cerca de $9,0 \mathrm{~cm}$ de diâmetro. O terceiro paciente realizou à época radiografia do abdome que mostrou elevação da hemi-cúpula diafragmática esquerda e imagem hidro-aérea subjacente; além disso foi também submetido a estudo contrastado do estômago, o qual se apresentava comprimido e rechaçado medialmente por massa localizada no hipocôndrio esquerdo (Figura 1).

A hemocultura foi negativa nos dois pacientes recentes. A cultura do material obtido do AE durante a operação identificou a presença da Salmonella enteritidis nestes mesmos pacientes, entretanto, mostrou-se negativa no terceiro paciente.

Os três pacientes foram submetidos à esplenectomia com drenagem da loja esplênica; o exame anatomopatológico do baço confirmou tratar-se de AE (Figura 2). A antibioticoterapia compreendeu a associação de ciprofloxacina, metronidazol e ampicilina para um dos pacientes, ceftriaxona e metronidazol para outro e cloranfenicol para o terceiro. O tempo de internação pós-operatória foi de 14 para um dos pacientes e de 18 dias os outros dois. Como intercorrência observou-se íleo pós-operatório em um dos casos e infecção da ferida cirúrgica em outro. Após a alta os dois pacientes mais recentes foram acompanhados em regime ambulatorial durante seis e treze meses; destes, o mais jovem foi re-internado no sexto mês por infarto agudo do miocárdio, enquanto o outro se manteve bem durante todo o período de acompanhamento.

\section{DISCUSSÃO}

Tendo em vista sua raridade, a discussão a cerca do AE geralmente está limitada a relatos de caso ou a pequenas séries institucionais. Os relatos de AE feitos entre 1900 e 1977, 1977 e 1986 e entre 1987 e 1995 mostram uma variação significativa entre os patógenos causadores da doença a partir de diferentes regiões geográficas associado a um amplo espectro de características demográficas e clínicas. As modificações no estilo de vida com conseqüente aumento da prevalência de doenças como a diabete mellitus, o câncer, o trauma, a imunossupressão decorrente de determinadas modalidades terapêuticas, a SIDA e até mesmo o uso crescente de drogas ilícitas por via endovenosa, modificaram os padrões clínicos do AE.

A incidência atual do AE é de difícil estimativa. Estudos de necrópsia no passado relatavam a presença do AE em 

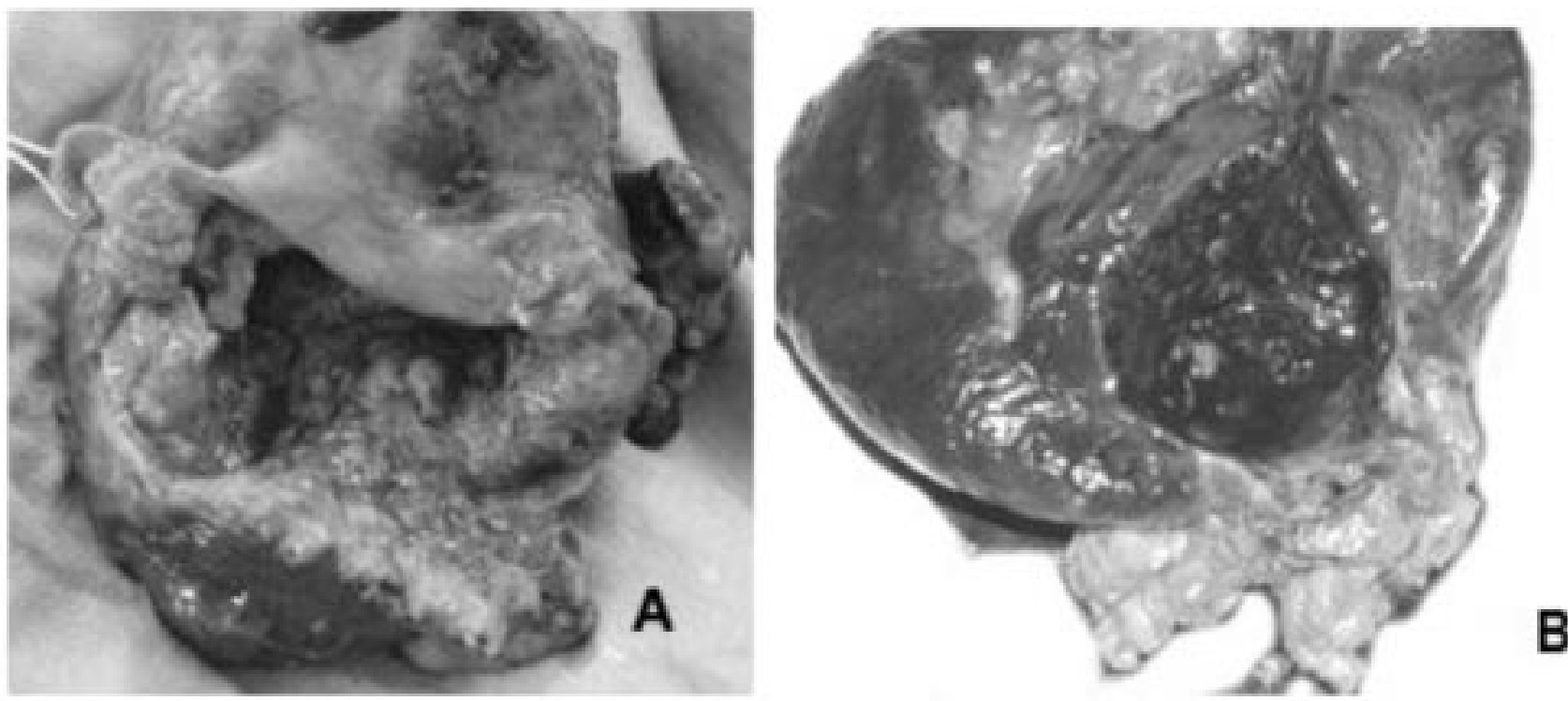

Figura 2 - Em A, baço quase totalmente substituído pela cavidade do abscesso; em B é possível observar solução de continuidade na superfície esplênica relacionada à cavidade do abscesso e segmento do grande omento responsável pelo bloqueio deste defeito.

$0,14 \%$ a $0,7 \%$, enquanto dados mais recentes referem ocorrência do AE em de 0,0049\% de mortes hospitalares / ano. Na prática clínica a incidência estimada é da ordem de $0,012 \%$ / 1000 internações / ano ou de 0,056\% / 1000 altas / ano ${ }^{1-3,8-}$ $10,12,14$. De forma condizente com estes dados, no período compreendido entre janeiro de 2001 a dezembro de 2005, foram diagnosticados e tratados apenas dois $(0,04 \%)$ únicos casos de $\mathrm{AE}$ entre os 4823 pacientes atendidos na Clínica Cirúrgica "A" do Hospital Universitário Gaffrée-Guinle. Ambos tiveram como agente causal a Salmonella enteritidis. O terceiro caso por nós estudado trata-se de paciente atendido por um dos autores (AMM) há cerca de 40 anos em outro nosocômio, época em que já era observado declínio da febre tifóide em nosso meio.

O AE como complicação da febre tifóide, da mesma forma que esta, apresentou declínio a partir dos anos 40 até 1980. A partir de então, houve aumento da ocorrência de outros tipos de sorotipo de Salmonella, coincidindo com o aumento no número de AE associado a Salmonella sp, de modo que este micro-organismo foi o isolado em até $20 \%$ dos $\mathrm{AE}$ relatados na literatura médica ${ }^{1,2,5,6,8-11,15,16}$. É digna de nota a ocorrência rara de AE mesmo em países em desenvolvimento, onde é alta a incidência de febre tifóide e/ou salmonelose na população geral ${ }^{5}$. Contudo, alguns autores acreditam que estas cifras podem ser subestimadas em áreas endêmicas de febre tifóide ${ }^{16}$.

É descrita, como aqui observada, a predominância pelo sexo masculino da ordem de $67 \%$, ou seja, uma relação de 2:1 entre homem e mulher. A média de idade dos pacientes com AE é de 41 anos, variando de seis meses a $90 \operatorname{anos}^{1,2,5}$.

Fatores predisponentes para o aparecimento do AE incluem hemoglobinopatias, trauma, bacteremia (especialmente aquelas associadas à endocardite), infecção em área contígua ao baço, qualquer condição que favoreça o enfarte esplênico, o uso endovenoso de drogas ilícitas e eventos que predispo- nham o estado de imunossupressão. Nos últimos anos, estas duas últimas condições têm se mostrado como elemento de importância relevante na gênese do $\mathrm{AE}^{1,2,5-7,11-14}$. Os pacientes aqui estudados eram previamente saudáveis e em nenhum deles foi possível identificar qualquer fator predisponente ao aparecimento do AE, quer durante a internação, quer ao longo do acompanhamento ambulatorial pós-operatório. Contudo, chama a atenção o fato destes pacientes terem desenvolvido a doença após viagem às regiões norte e nordeste do país. De modo distinto ao observado no presente relato, não foi identificada a ocorrência de AE em 6502 casos de salmonelose e em 2000 casos de febre tifóide envolvendo indivíduos previamente saudáveis ${ }^{5}$. A presença de possíveis causas de predisposição ao AE causado por Salmonella esteve presente em $40 \%$ dos pacientes de um estudo multicêntrico realizado na América Latina ${ }^{5}$.

O diagnóstico do AE é difícil quando baseado exclusivamente na história e no exame físico. A tríade representada por febre, dor no quadrante superior esquerdo e leucocitose é sugestiva, mas não patognomônica do $\mathrm{AE}^{1-3,5,6}$. Os achados clínicos e laboratoriais incluem febre (84-93\%), leucocitose (60-88\%), dor abdominal difusa (58\%), e/ou em quadrante superior esquerdo do abdome (39-50\%), sinais de irritação peritoneal difusa por todo o abdome $(60 \%)$ ou no quadrante superior esquerdo (38\%), esplenomegalia (31-56\%), dor pleurítica (14\%), derrame pleural esquerdo (22\%), dor no ombro esquerdo (4\%), síndrome toxêmica (15\%) e vômitos (14\%), leucocitose $(60 \%)$ e trombocitose $(10 \%)^{1-3,6,7,11,12,14}$. Em $20 \%$ dos pacientes cujo o micro-organismo isolado foi a Salmonella havia presença de diarréia ${ }^{5}$. Os pacientes aqui relatados apresentaram manifestações clínicas e laboratoriais observadas com certa freqüência no $\mathrm{AE}$, como grande parte das acima descritas, o que permitiu aventar clinicamente a hipótese diagnóstica de AE.

Como observado no presente relato, o AE causado por Salmonella, invariavelmente (90\%) é solitário, de 
forma que apenas aqueles associados a condições de imunodeficiência ou bacteremia sustentada, como na endocardite, tem maior propensão a serem múltiplos ${ }^{5}$.

$\mathrm{O}$ estudo microbiológico de amostras de sangue e do material obtido diretamente do abscesso costuma apresentar taxas distintas de positividade. A hemocultura é capaz de identificar o micro-organismo em 14 a $70 \%$ dos casos ${ }^{15}$; Ooi e Leong ${ }^{1}$ consignaram a este método uma positividade $48 \%$ nos pacientes estudados, mas em somente $23 \%$ deles houve concomitância entre os resultados obtidos na hemocultura e no material do abscesso. Em contraste, $73 \%$ das culturas do material recolhido do AE foram capazes de identificar o agente causal ${ }^{1}$. Torres no estudo de dez casos de AE causados por Salmonella, obtive cultura positiva a partir do material do abscesso em seis casos e a partir do sangue em cinco pacientes, sendo que em três destes o sangue foi a única fonte de positividade; em dois outros foi possível isolar a Salmonella também nas fezes ${ }^{5}$. Neste mesmo estudo, os autores identificaram a S. tiphy em $60 \%$ dos pacientes, enquanto nos demais o agente responsável foi a $S$. enteritidis ${ }^{5}$. Ghadage e Bal ${ }^{15}$ isolaram a Salmonella enteritidis sorotipo Worthington tanto na hemocultura quanto no material do AE. Em dois casos do presente relato, nos quais foi realizada a hemocultura, a mesma mostrou-se negativa, enquanto a cultura do material obtido diretamente do abscesso foi capaz de identificar em ambos a $S$. enteritidis como o único agente causal.. De forma habitual, há crescimento de apenas um micro-organismo, apenas em cerca de $11 \%$ dos pacientes a flora é polimicrobiana $^{1}$. O número significativo de casos em que ocorre dissonância do micro-organismo isolado na hemocultura daquele presente no material do AE, pode fazer difícil a escolha do antibiótico a ser empregado até que o teste de sensibilidade esteja pronto.

$\mathrm{O}$ advento dos exames de imagem como a US e a TC, veio permitir não só que a suspeição de tais achados fosse mais freqüente além de contribuir para melhor afirmação, tornando-se assim, exames cruciais para o diagnóstico do $\mathrm{AE}^{16}$. A sensibilidade da TC é da ordem de $96 \%$, enquanto para a US é de $87 \%^{1-4}$. Embora a US ofereça menor custo e tenha uma sensibilidade próxima a da TC, esta última se presta melhor ao diagnóstico dos abscessos intra-abdominais em geral e do AE em particular ${ }^{1,4,5,7}$. Os achados usuais da US estão representados por lesões do parênquima esplênico de característica anecóica ou hipoecóica, algumas vezes com bordas irregulares e limites mal definidos, conteúdo não homogêneo e a presença de debris ${ }^{1,16}$. A TC permite a identificação de lesões de alguns milímetros de diâmetro e a localização anatômica precisa do AE. A imagem típica é representada por lesão parenquimatosa de baixa densidade com captação periférica após injeção intravenosa de contraste; pode ser encontrados ainda nível hidro-aéreo e extensão extraesplênica do abscesso. Pequena quantidade de gás é melhor observada pela TC do que pela $\mathrm{US}^{1,3-5}$. Em dois dos casos aqui descritos os achados quer da US, quer da TC, eram compatíveis com aqueles encontrados no AE. O terceiro caso foi explorado à época pela radiologia convencional, cujos achados descritos eram sugestivos de AE. Os resultados obtidos com a radiografia do tórax e com a radiografia simples do abdome são sugestivos de AE em cerca de somente $30 \%$ dos pacientes ${ }^{1}$.

Tradicionalmente o tratamento do AE inclui a antibioticoterapia endovenosa e a esplenectomia. Recentemente, a drenagem per-cutânea (DPC) guiada por US ou TC vem ganhando espaço em pacientes selecionados, podendo alcançar nestes casos índices de sucesso que variam de $51 \%$ a $100 \%{ }^{2-4}$, resultados estes semelhantes à taxa de resolução da esplenectomia que é de $100 \%{ }^{1}$. A DPC alcança maiores possibilidades de sucesso quando o AE é único, sem septações em seu interior e o conteúdo liquido é fluido de forma a permitir uma boa evacuação, a lesão é de situação periférica e localizado no 1/3 médio ou no pólo inferior do baço. Nesta circunstância, a DPC pode ser o procedimento inicial de esco$\mathrm{lha}^{4}$. Nos abscessos múltiplos ou multiloculares, com septações e debris, na presença de cavidade mal definida ou muito pequena, a localização central no parênquima esplênico ou a existência de coleção peri-esplênica, deve-se optar pela esplenectomia ${ }^{1,4}$. No insucesso da DPC é possível realizar a remoção do baço sem aumento nas taxas de morbi-mortalidade ${ }^{1}$. Quando o micro-organismo é identificado pela hemocultura e a coleção é pequena, é possível empregar a terapia antimicrobiana de forma prolongada acompanhando a evolução do AE pela US ou TC periódica ${ }^{2,3,5}$. É descrito por alguns autores o emprego de tratamento conservador, representado exclusivamente pela antibioticoterapia prolongada, em dois pacientes jovens com grande AE solitário causados pela $S$. typhi. ${ }^{5}$. Entretanto, nenhum outro relato semelhante foi encontrado. Há de ser considerado o fato da resistência a múltiplos antibióticos, inclusive cefalosporinas de terceira geração, por parte de diferentes sorotipos de Salmonella não typhi ${ }^{15}$. Nos casos ora apresentados, a DPC não foi indicada por conta do fleimão e da alteração do tempo e da atividade da protrombina em um dos casos, bem como pela presença de debris e de material sólido no interior do abscesso e da localização polar superior em íntima relação com o diafragma esquerdo em outro. À época em que foi tratado o terceiro paciente deste relato, a DPC ainda não era um método empregado para tal circunstância.

A taxa de mortalidade varia de 0 a $14 \%$, podendo alcançar a cifra de $25 \%$ em pacientes imunodeprimidos ${ }^{1,2,4,5}$. A mortalidade relacionada à esplenectomia como forma inicial de tratamento é de $17 \%$, já quando é realizada após falha da DPC é de $14 \%{ }^{1}$. A aspiração ou a DPC apresentam taxa de mortalidade de $3,2 \%$ e $0 \%$ respectivamente, entretanto, isto pode refletir tão somente a seleção de casos menos complexos para receberem estes tipos de tratamento. A taxa de sobrevida de pacientes com AE causado por Salmonela tratado pela esplenectomia associada a antibioticoterapia é de $87 \%$ a $90 \% 5$

Em resumo, o AE é uma doença pouco freqüente, em especial quando causado por Salmonella, ainda é de difícil identificação e potencialmente fatal, em especial, quando o diagnóstico e a terapêutica adequada não são efetuados precocemente. 


\begin{abstract}
Background: Splenic abscess is a uncommon disease and remains a diagnostic challenge. Outcome is fatal when the condition is not promptly recognized and treated. The aim of this study was to analyze the demographic, clinical, diagnostic methods and management characteristic of patients with salmonelal splenic abscess. Methods: Retrospective study at General and Digestive Surgery Service - Hospital Universitário Gaffrée-Guinle of the Universidade Federal do Estado do Rio de Janeiro - UNIRIO from January 2001 to December 2005 resulting in a total number of 4823 patients has been performed. An additional case treated by one author was added. Results: During the studied period two cases of splenic abscess due to Salmonella enteritidis was treated; a third older case was due to Salmonella typhi. All patients were man; the mean age was 45 years, all were immunocompetent and had large solitary lesion. All patients underwent splenectomy and antibiotic therapy; the outcome was favorable for all patients. Conclusions: Nowadays still is possible that splenic abscess is due to Salmonella species etiology; typically, all patients are immunocompetents adult males and large solitary lesions within necrotic material. In this condition, splenectomy and intravenous antibiotic administration appear to constitute the most convenient therapy.
\end{abstract}

Key words: Spleen; Abscess; Splenic diseases; Salmonella infections; Splenectomy.

\section{REFERÊNCIAS}

1. Ooi LL, Leong SS. Splenic abscesses from 1987 to 1995 . Am J Surg. 1997; 174(1):87-93.

2. de Bree E, Tsiftsis D, Christodoulakis M, Harocopos G, Schoretsanitis G, Melissas J. Splenic abscess: a diagnostic and therapeutic challenge. Acta Chir Belg. 1998;98(5):199-202.

3. Ng KK, Lee TY, Wan YL, Tan CF, Lui KW, Cheung YC, Cheng YF. Splenic abscess: diagnosis and management. Hepatogastroenterology. 2002; 49(44):567-71.

4. Thanos L, Dailiana T, Papaioannou G, Nikita A, Koutrouvelis H, Kelekis DA. Percutaneous CT-guided drainage of splenic abscess. AJR Am J Roentgenol. 2002;179(3):629-32.

5. Torres JR, Gotuzzo E, Istúriz R, Elster C, Wolff M, Northland $\mathrm{R}$, Christenson B, Clara L. Salmonellal splenic abscess in the antibiotic era: a Latin American perspective. Clin Infect Dis. 1994;19(5):871-5.

6. Lee CH, Leu HS, Hu TH, Liu JW. Splenic abscess in southern Taiwan. J Microbiol Immunol Infect. 2004;37(1):39-44.

7. Martins ACA, Vieira LFDF, Ferraz AAB, Santos Jr MA, Ferraz EM. Abscesso esplênico: mudanças nos fatores de risco e nas opções de tratamento. Rev Col Bras Cir. 2005;32(6):337-41.

8. Saimot G, Aouati A, Delaitre B, Bensebaa A. Abcès splénique à Salmonella thyphi para A. Nouv Presse Med. 1979;8(46):3835-7.

9. Kumar KHR, Kate V, Ananthakrishnan N, Sharma AK, Kanungo R, Badrinath S, Ravikumar KH, Reba K. Splenic abscess due to Salmonella infection. Indian J Med Microbiol. 1996;14(2):109-11.

10. Sarr MG, Zuidema GD. Splenic Abscess - presentation, diagnosis, and treatment. Surgery. 1982;92(3):480-5.
11. Nelken N, Ignatius J, Skinner M, Christensen N. Changing clinical spectrum of splenic abscess. A multicenter study and review of the literature. Am J Surg. 1987;154(1):27-34.

12. Paris S, Weiss SM, Ayers WH, Clarke LE. Splenic abscess. Am Surg. 1994;60(5):358-61.

13. Herman P, Oliveira e Silva A, Chaib E, D'Albuquerque LC, Pugliese V, Machado MC, Pinotti HW. Splenic abscess. Br J Surg. 1995;82(3):355.

14. Phillips GS, Radosevich MD, Lipsett PA. Splenic abscess: another look at an old disease. Arch Surg. 1997;132(12):1331-5; discussion 1335-6.

15. Ghadage DP, Bal AM. Isolation of Salmonella enterica serotype Worthington from a splenic abscess in a patient with chronic myeloid leukemia. Braz J Infect Dis. 2002;6(2):88-90.

16. Allal R, Kastler B, Gangi A, Bensaid AH, Bouali O, Cherrak C, Brun F, Dietemann JL. Splenic abscesses in typhoid fever: US and CT studies. J Comput Assist Tomogr. 1993;17(1):90-3.

Como citar este artigo:

von Glen Herkenhoff B, Ferreira LGS, Cavedagne M, Salomão RN, Maia AM, Iglesias AC. Abscesso esplênico por Salmonella. Rev Col Bras Cir. [periódico na Internet] 2006 Mai-Jun; 33(3). Disponível em URL: http://www.scielo.br/rcbc

Correspondência:

Antonio Carlos Iglesias

Rua Jorge Rudge 120, Bloco 2, Apto. 1007

20550-220 - Rio de Janeiro - RJ

E-mail: iglesias@momentus.com.br 